\title{
In vitro and in vivo anti-diabetic properties of Hibiscus sabdariffa
}

\author{
S. Zulfiqar ${ }^{1}$, K. Benton ${ }^{1}$, T. Hassan ${ }^{1}$, L. Marshall ${ }^{1}$ and C. Boesch ${ }^{1}$ \\ ${ }^{1}$ School of Food Science and Nutrition, Nutritional Sciences and Epidemiology, University of Leeds, LS2 9JT, Leeds, \\ $U K$.
}

Hibiscus sabdariffa ( $H$. sabdariffa), also known as roselle or red tea, is a member of the Malvaceae family, rich in anthocyanins and other bioactive compounds, which has been associated with a number of health benefits such as lowering of blood pressure and plasma cholesterol $^{(1)}$. The intake of $H$. sabdariffa has also been linked to prevention of diabetes, however, there is limited evidence on effects of $H$. sabdariffa on postprandial glycaemia and/or chronic markers of glycaemic control. A number of polyphenols have demonstrated potential in reducing blood glucose levels in an acute manner through modulation of postprandial glycaemic response when consumed with a carbohydrate source such as high starch foods, glucose and/or sucrose ${ }^{(2)}$.

The aim of our project was to investigate the in vitro inhibitory properties of $H$. sabdariffa on $\alpha$-glucosidase enzyme activity and to confirm the findings in an in vivo glycaemic response study. Following incubation with different concentrations $(0,2.5-25 \mathrm{mg} / \mathrm{ml})$ of aquaous extracts of $H$. sabdariffa, the activity of $\alpha$-glucosidase was determined via spectrophotometer using p-nitrophenyl- $\alpha$ D-glucopyranoside as substrate following a standard protocol. Acarbose was used as positive control and data are expressed as per cent inhibition. For the in vivo study, eight healthy volunteers (3 male and 5 females) were recruited, aged 19-23 years, who were asked to consume $400 \mathrm{ml}$ of either hot water or hibiscus tea alongside a portion of white bread to provide $50 \mathrm{~g}$ available carbohydrates. The capillary blood was collected via finger prick in regular intervals following tea and bread consumption $(0,15,30,45,60$, $90,120,150 \mathrm{~min})$. Blood glucose levels were determined using glucometer. Two-tailed paired t-test was used to analyse the data.

The results from in vitro experiments demonstrate that $H$. sabdariffa preparations dose-dependently inhibited $\alpha$-glucosidase activity with an $\mathrm{IC}_{50}$ of $10 \mathrm{mg} / \mathrm{ml}$ extract (equivalent to $55.7 \mathrm{mg} / \mathrm{ml}$ anthocyanins).

Overall, the in in vivo data (Fig. 1) do not show any differences following the intake of hibiscus tea in comparison to control (hot water) alongside white bread over the course of the $150 \mathrm{~min}$ experiment. The lack of effect on glucose response may be related to insufficient local concentrations of hibiscus but awaits further confirmation in a larger sample size.

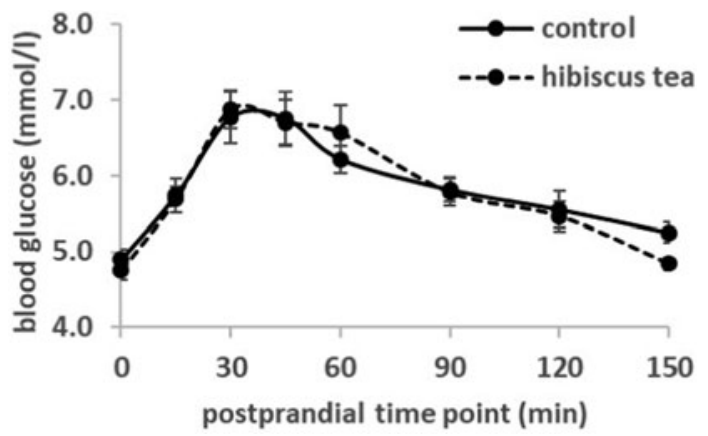

Fig 1. Blood glucose concentration at baseline and following postprandial phase over $150 \mathrm{~min}$. Values represent mean with SE of eight participants. No significant differences were determined.

Our data indicate, that, although, in vitro experiments indicate the potential of $H$. sabdariffa to reduce carbohydrate digestion, under in vivo conditions, the acute consumption of $H$. sabdariffa might not relevant to reduce postprandial glucose response.

1. Hopkins AL, Lamm MG, Funk J, Ritenbaugh C (2013) Fitoterapia 85, 84-94.

2. Coe S \& Ryan L (2016) J Nutr Sci 5, e24. 